\title{
An experimental setup for nondestructive deposition of size-selected clusters
}

\author{
R. Klingeler, P. S. Bechthold, M. Neeb, a) and W. Eberhardt \\ Institut für Festkörperforschung, Forschungszentrum Jülich GmbH, 52425 Jülich, Germany
}

(Received 20 July 2001; accepted for publication 7 January 2002)

\begin{abstract}
An experimental setup for the deposition of mass-selected clusters using a laser vaporization source and a magnetic field mass selector is presented. Nondestructive deposition and a coverage of $1 \%$ of a monolayer within $5 \mathrm{~h}$ are achieved for mass-selected metallofullerene clusters as demonstrated for deposited Ce@ $\mathrm{C}_{60}$ on highly oriented pyrolytic graphite. (c) 2002 American Institute of Physics.
\end{abstract}

[DOI: $10.1063 / 1.1455135]$

\section{INTRODUCTION}

One of the main interests in cluster physics is the atomby-atom investigation of material properties when going from the atom towards the bulk. ${ }^{1}$ For example, the monitoring of the band gap closure in $\mathrm{Hg}$ clusters as a function of cluster size has been a milestone in cluster physics. ${ }^{2}$ So far, most experiments on mass-selected clusters are limited to the gas phase, e.g., mass spectroscopy, ${ }^{3,4}$ ion mobility measurements, ${ }^{5,6}$ and laser photoelectron spectroscopy on anions. ${ }^{7,8}$ While the study of clusters in the gas phase provides invaluable information on isolated systems the investigation of supported clusters is essential for almost any application.

One of the main motivations is a microscopic understanding of chemical reactions and catalytic processes. ${ }^{9}$ There is a worldwide activity to develop more efficient and, particularly, more selective catalysts in order to enhance their chemical efficiency. For a systematic investigation the electronic structure and geometry of individual clusters must be explored, e.g., to analyze the chemically active centers of the cluster, the interacting orbitals, the vibrational frequencies, bond orders and bond strengths, and the valence electron density. Particularly the cluster-surface interaction needs to be carefully examined because the substrate might totally reshape the geometry and electronic structure of the cluster. On surfaces additional spectroscopic tools become available, like photoelectron and photoabsorption spectroscopy using $\mathrm{UV} / \mathrm{vis}$ radiation and $\mathrm{x}$ rays or infrared and Raman spectroscopy. ${ }^{10-17}$ Essential information on charge transfer, density of states, orbital interaction, hybridization, chemical information, Jahn-Teller distortion, and intermolecular cluster interaction are revealed from these techniques. Furthermore, using scanning tunneling spectroscopy the density of states both below and in particular above the Fermi level can be monitored. ${ }^{18-21}$ The latter information is not available from gas phase experiments. Moreover, the gradual changes of the properties from individual clusters to two- and threedimensional cluster assemblies as well as the properties of clusters on different substrates could be spectroscopically

${ }^{a)}$ Electronic mail: m.neeb@fz-juelich.de studied upon size-selective deposition. ${ }^{22}$ Finally, gathering all the information could be used to tailor new nanoscopic materials with unique chemical, magnetic, or metallic properties. In order to apply surface sensitive techniques a cluster density of about $1 \%$ of a monolayer corresponding to $\sim 1$ cluster per $100 \mathrm{~nm}^{2}$ is needed. These challenging objectives require extremely intense cluster sources combined to high transmission mass spectrometers. Furthermore, the landing of mass-selected clusters on the surface needs to be as soft as possible to reduce the distortions of the clusters and surfaces to a minimum.

So far, different cluster deposition setups regarding the type of cluster source and mass selection have been realized. The chemical reactivity of size-selected supported Pt clusters has been investigated by Heiz et al. ${ }^{9}$ These samples have been produced using a laser vaporization source and a quadrupole mass selector. ${ }^{23}$ Quantum confinement effects of sizeselected supported Pt clusters have been measured by the Meiwes-Broer group using scanning tunneling spectroscopy. ${ }^{24}$ They produced the clusters in an arc discharge source, the mass selection has been achieved using a magnetic sector field. The same combination has been applied by the Gantefor group for x-ray spectroscopy on sizeselected supported Al clusters. ${ }^{25}$ A time-of-flight mass spectrometer has been adapted to an arc discharge cluster ion source by Kaiser $e t a l$. in order to investigate the mobility of $\mathrm{Sb}$ clusters on surfaces using scanning tunneling microscopy. ${ }^{26}$ The above experiments take advantage of the fact that laser vaporization and arc discharge sources are capable of producing clusters of metals and semiconductors that are hard to vaporize. They usually provide good conditions for cluster aggregation. An adiabatic expansion of the cluster-helium mixture provides ions with a narrow kinetic energy distribution which are then mass-selected. Due to this narrow energy distribution a magnetic or quadrupole mass selection has the advantage of an optimal control over the deposition energy within $\sim 1 \mathrm{eV}$. The overall transmission is usually better for the gridless time-of-flight method, yet it has to overcome problems arising from the spatial extension of the cluster beam.

Principally different from the soft-landing method via electrostatic deceleration fields is the deposition into a rare 


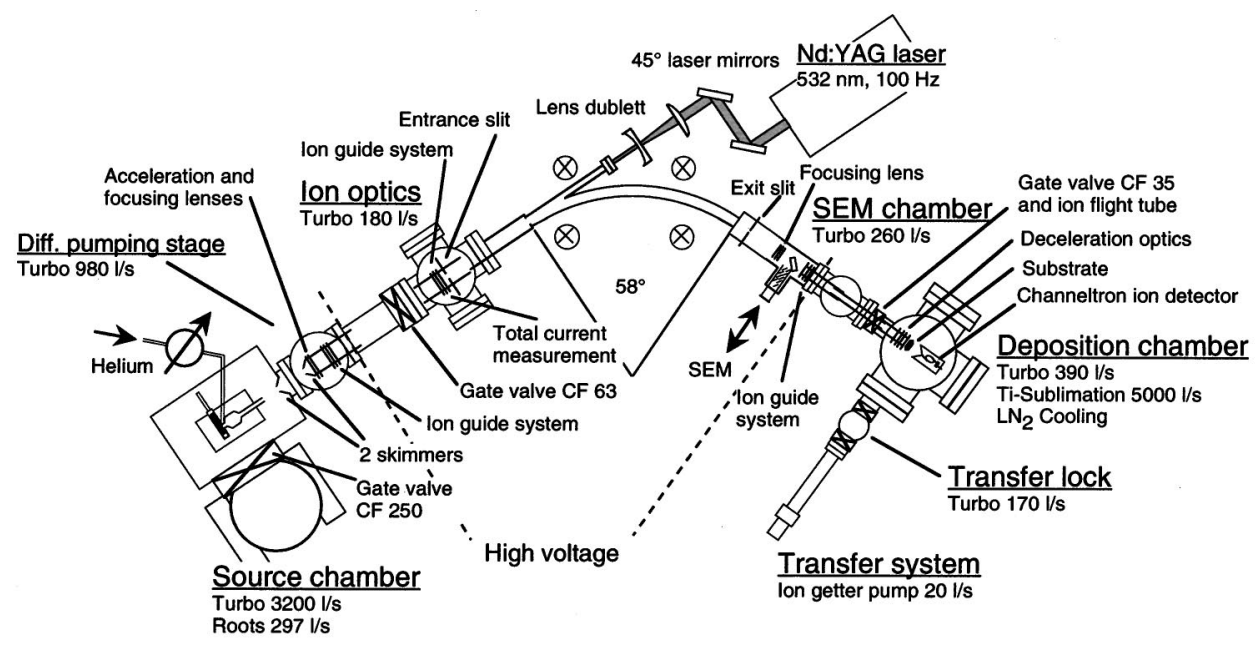

FIG. 1. Sketch of the cluster deposition machine. Three main components are the cluster source to the left, the magnetic mass analyzer in the middle, and the deposition stage to the right.

gas matrix at low temperatures. This method has been successfully employed by Lau et al. who have investigated the electronic structure of small $3 d$ metal clusters using synchrotron radiation. ${ }^{27,28}$ Their clusters are produced via ion sputtering which principally lacks the advantage of cluster condensation inherent to the other sources. The advantage is higher cluster intensity, especially for small ones. Matrix deposition from a sputter source along with a quadrupole mass selection has also been used by Harbich et al. in order to perform scanning tunneling microscopy (STM) and fluorescence spectroscopy on small Ag clusters. ${ }^{29,30}$ Very recently, von Issendorf et al. have reported on the possibility of soft cluster deposition combining a magnetron sputtering source and a newly developed time-of-flight mass spectrometer. $^{31}$

In the present article a cluster deposition machine for soft landing is described, which combines a pulsed laservaporization source and a magnetic cluster ion selector. ${ }^{32}$ The performance of the machine is illustrated by mass spectra of metal-carbon clusters and STM pictures of $\mathrm{Ce} @ \mathrm{C}_{60}$ on highly oriented pyrolytic graphite (HOPG).

\section{EXPERIMENTAL SETUP}

Figure 1 shows an overview of the experimental setup. The machine combines a laser-vaporation source, a magnetic field mass selector, and a soft-landing deposition stage. The clusters are deposited at UHV conditions. Samples can be loaded into various experiments without interrupting the UHV conditions using an UHV transport chamber.

\section{A. Cluster source}

Our design follows the one proposed by Heiz et al. ${ }^{23}$ The principles of a laser-vaporation source are described elsewhere. ${ }^{33}$ It has been modified in order to gain high ion currents of doped fullerenes along with a long-term stability of the cluster source. Target rods with diameters between 6 and $15 \mathrm{~mm}$ and lengths of $50 \mathrm{~mm}$ are used. During operation the rod rotates at a variable frequency of typically $0.2 \mathrm{~Hz}$, and the rotation is superimposed by a translation in order to avoid hole burning.

The laser beam is fed into the source on the axis of the outgoing cluster beam, which gives the advantage of a tighter source since no additional channel is needed. The focusing lens doublet is located about $2 \mathrm{~m}$ away from the target rod. A plano-convex and a plano-concave lens with focal lengths of 300 and $-200 \mathrm{~mm}$ have been used, respectively. In order to achieve a small beam spot on the sample the requirements to the laser beam divergence are essential. The second harmonic $(532 \mathrm{~nm})$ of a $Q$-switched Nd:YAG laser (Coherent Infinity 40-100) has been used as the vaporization laser. The $0.7 \mathrm{mrad}$ beam divergence allows excellent focusing conditions. The laser pulse energy is typically set to $20 \mathrm{~mJ}$ during operation. Higher ion currents were achieved when the target rod is slightly out of focus.

To increase cluster condensation, a thermalization chamber with $10 \mathrm{~mm}$ diameter and $12 \mathrm{~mm}$ length is used. For most efficient fullerene production, this is followed by a drift tube of $20 \mathrm{~mm}$ length with a nozzle of $3 \mathrm{~mm}$ diameter. The helium inlet nozzle ( $1 \mathrm{~mm}$ diameter, $6 \mathrm{~mm}$ length) is directed onto the laser spot on the target rod, and it can be either pulsed or continuous. In the pulsed operation mode, a solenoid valve (General Valve, series 9) is used which is driven by a homemade power supply at $220 \mathrm{~V}$. A variable opening time between 30 and $600 \mu$ s is possible, and the optimal source performance is achieved for opening times of 90-140 $\mu$ s depending on the helium pressure applied and the desired fullerene. The $Q$-switch of the laser is the master trigger of the whole system. Using a built-in pretrigger a typical delay time of $510 \mu$ s between the helium inlet and the laser shot is applied. The helium (He 6.0) is injected into the system at backing pressures ranging between 0.5 and 20 bar. In the continuous operation mode proper cluster condensation is only possible when the helium pressure is adjusted to $\sim 2$ mbar in the source chamber. While the pulsed mode of operation supplies slightly higher ion yields, the continuous mode guarantees a more stable overall performance of the source. Before entering the mass spectrometer the molecular beam is skimmed twice passing a differential pumping stage. The diameter of the skimmers is $5 \mathrm{~mm}$ each. The naturally ionized clusters present in the cluster beam are accelerated to $4 \mathrm{keV}$ kinetic energy prior to mass selection.

While for metal targets, e.g., Pt, the evaporation of matter is almost negligible yielding a quite stable source perfor- 
mance and high target lifetimes in the range of $1000 \mathrm{~h}$, the graphite or mixed metal-graphite targets burn more rapidly. In this case, the lifetime of the target is limited to $\sim 10 \mathrm{~h}$, and the substantial removal of matter from the target rod leads to an unstable cluster source performance on a time scale of several minutes. Moreover, the deposition of carbon soot inside the cluster source generally blocks the nozzle after some time which usually needs to be cleared hourly. The maximum deposition rate was achieved at a background pressure of 2-4 bar He and an opening time of the pulsed valve of 90-140 $\mu$ s. A background pressure of less than 1 bar has been of advantage to produce larger metallofullerene clusters. The pressure in the cluster source chamber was usually held within the $10^{-4}$ mbar range during deposition.

\section{B. Mass selection}

For mass selection a magnetic field analyzer (AMD Intectra) is used. The mass resolution can be pushed to a maximum $m / \Delta m \approx 1000$, but for the sake of higher transmission the system has been operated at $m / \Delta m \approx 300$ during deposition. The overall transmission of the mass selector has been determined to be $60 \%$ for $\mathrm{Cs}^{+}$cations produced by a cesium oven. One of the main requirements for nondestructive deposition is the energy control of the incoming cluster ions. As the width of the kinetic energy distribution of the monodispersed cluster ion beam is typically about $1 \mathrm{eV}$ when using a magnetic sector field, a proper deceleration prior to deposition is possible.

The cations in the expanding cluster beam are accelerated to $4 \mathrm{keV}$ kinetic energy immediately behind the second skimmer. In order to minimize the ion loss the field lines of the accelerating field end on the surface of the skimmer. The accelerated ions are focused into the entrance slit of the magnetic sector field and are deflected by $58^{\circ}$ in a field with a magnetic flux density up to $1.2 \mathrm{~T}$. An electron multiplier behind the exit slit enables us to record mass spectra by scanning the magnetic flux density of the deflection field at constant acceleration voltage.

\section{Cluster deposition}

At a constant magnetic flux density a certain cluster mass is selected to pass the exit slit of the mass selection stage and to enter the deceleration optics. The $4 \mathrm{keV}$ kinetic energy of the ions is reduced using three subsequent electrostatic lenses. The cluster beam is focused onto a circular aperture of $3 \mathrm{~mm}$ diameter located about $1 \mathrm{~mm}$ in front of the substrate. As the cluster source is connected to ground potential, the voltage applied to the substrate determines the deposition energy of the ions. In order to combine the lowest possible deposition energy with a minimum ion loss due to stray fields, the substrate voltage is adjusted according to the mass of the selected cluster ions. After adiabatic expansion in helium the clusters have a narrow velocity distribution about a mean value of $1600 \mathrm{~m} / \mathrm{s}$. Clusters with different masses have different kinetic energies. For example, when depositing $\mathrm{Ce} @ \mathrm{C}_{60}{ }^{+} \quad($ mass=861 amu $)$ onto a grounded substrate, the kinetic energy of the cluster is $11 \mathrm{eV}$ resulting in a kinetic energy per atom of $180 \mathrm{meV}$. For fullerenes this allows a nondestructive cluster deposition. The ion current on the substrate is measured using a Keithley analog electrometer. When removing the sample the ions can also be monitored using a channeltron that is located right behind the substrate.

Highly oriented pyrolytic graphite (HOPG) was mainly used as substrate, which is a standard surface in scanning tunneling experiments. Moreover, it is inert with respect to rest gas contamination and is simple to prepare. Along with its electrical conductivity it is a very suitable substrate for cluster deposition. At low coverage, the substrate for x-ray photoelectron spectroscopy must be chosen in a way that the cluster-related core level lines are not masked by any substrate peaks. The clusters are deposited at room temperature.

The cluster-covered samples can be transferred to the STM without interrupting the UHV conditions. Using a portable UHV suitcase they can be loaded into various other surface investigation machines such as X-ray photoelectron and IR spectrometers.

\section{Vacuum system}

In order to overcome the nine orders of magnitude in pressure between the cluster source pressure $(2 \mathrm{mbar})$ and the desired pressure in the deposition chamber $\left(10^{-9}\right.$ mbar $)$ five differential pumping stages are used. Moreover, the pressure in the source chamber must not exceed $10^{-3}$ mbar. Otherwise, the conditions are unfit for adiabatic expansion, and a scattering-free molecular beam cannot be formed. The source chamber is pumped by a $3200 \mathrm{l} / \mathrm{s}$ turbopump (Pfeiffer TPU 2200) backed by a 300 1/s roots blower (Pfeiffer WKP 1000 A) and a 36 1/s rotary vein pump (Pfeiffer DUO 120 A). When operating the cluster source at constant helium flow, the pressure in the source chamber is in the low $10^{-4}$ mbar range. Two skimmers with $5 \mathrm{~mm}$ diameter each border a chamber pumped by a $920 \mathrm{l} / \mathrm{s}$ turbo drag pump (Pfeiffer TMU $1001 \mathrm{D})$. The chambers containing the entrance and exit slits of the mass analyzer are pumped by a $180 \mathrm{l} / \mathrm{s}$ (Pfeiffer TPU 180) and a 220 1/s (Pfeiffer TMU 260) turbopump, respectively. The ion tube between the poles of the deflection magnet separates the ion source optics and the deposition optics efficiently. A low cross section $\left(\sim 5 \times 30 \mathrm{~mm}^{2}\right)$ of 50 $\mathrm{cm}$ length results in a high flow resistance. The pressure in the chamber immediately behind the magnet is about 5 $\times 10^{-8}$ mbar during operation. This chamber also contains the ion detector. The deposition chamber is pumped by a 390 1/s turbopump (Pfeiffer TPU $450 \mathrm{H}$ ), a titanium sublimation pump, and a liquid nitrogen trap. This chamber is separated from the previous chamber by an ion drift tube of $25 \mathrm{~mm}$ diameter and $350 \mathrm{~mm}$ length. The tube is mobile in order to allow the closure of a gate valve when not operating the deposition. Thus the contamination of the deposition chamber is limited, e.g., when recording mass spectra. During deposition the pressure is in the high $10^{-9}$ mbar range, mainly due to helium gas. The transfer system is equipped with a battery-driven ion getter pump in order to warrant UHV conditions during sample transfer. 


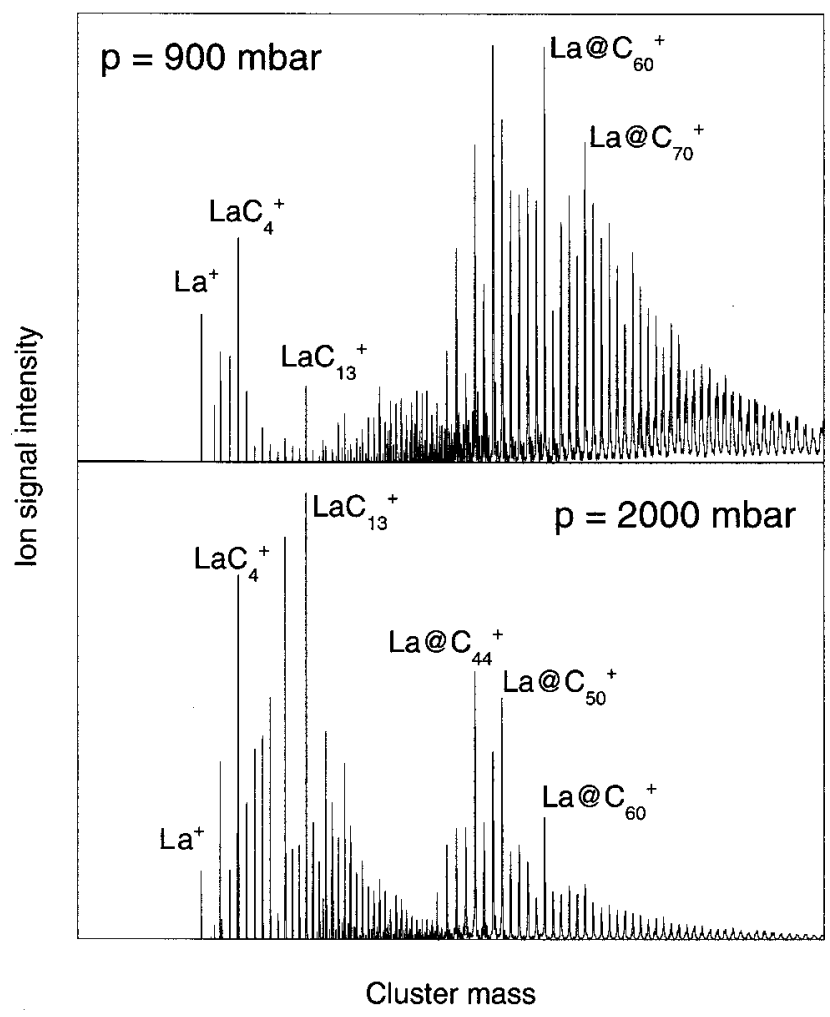

FIG. 2. Abundance spectra of lanthanum-doped carbon cluster cations taken at different source conditions. At low helium backing pressure (900 mbar), the size regime of doped fullerenes La@ $\mathrm{C}_{n}{ }^{+}(n \geqslant 36)$ is prominent. In contrast, at higher pressure (2000 mbar) $\mathrm{LaC}_{n}{ }^{+}$clusters with less than 20 carbon atoms gain intensity with respect to the low-pressure spectrum.

\section{RESULTS}

\section{A. Production of carbon clusters}

The cluster condensation process depends crucially on the applied helium pressure. A general trend can be observed: at low helium pressure the production of fullerenes and cage structures is preferred, whereas at higher helium pressure small clusters are produced preferentially. Figure 2 shows mass spectra of lanthanum-doped carbon cluster cations taken at different helium pressure conditions. As shown previously, the mass spectra obtained from metal-doped carbon rods (doping concentration 1\%, metal $=\mathrm{Sc}, \mathrm{Y}, \mathrm{La}, \mathrm{Ce}$, and Gd) usually start at the metal monomer. ${ }^{34,35}$ At low helium backing pressure (900 mbar), the abundance of the clusters in the cage regime $\mathrm{La} @ \mathrm{C}_{n}{ }^{+}(n \geqslant 36)$ is strongly enhanced with respect to smaller clusters. At a backing pressure of 2 bar the main intensity switches to lower masses, and $\mathrm{LaC}_{n}{ }^{+}$clusters with up to 20 carbon atoms gain intensity. At all pressures applied, $\mathrm{LaC}_{n}{ }^{+}$clusters with $n=0,2,3$, and 4 appear in the spectra, whereas clusters with $5 \leqslant n \leqslant 20$ disappear at still higher pressures.

\section{B. Mass resolution}

Figure 3 shows a mass spectrum of pure carbon cluster cations in the mass range up to $2714 \mathrm{amu}$. The onset of $\mathrm{C}_{n}{ }^{+}$ cluster intensity is found around $n=30$, which is also supposed to be the onset of cage structures. The overall distribution peaks around $n=140$, and clusters with more than 200 carbon atoms are produced in considerable amounts. As only even-numbered clusters show up in the spectrum, fullerenes are the most likely structures in the cluster beam. This spectrum has been recorded in the pulsed valve mode at a particularly low helium background pressure of $540 \mathrm{mbar}$ and moderate laser power. The inset shows an enlargement of the mass region between 1420 and $1550 \mathrm{amu}$. The peak width at half maximum is about 6 amu yielding a mass resolution of $m / \Delta m \approx 300$. This spectrum has been taken with both the entrance and exit slits wide open in order to get maximum ion throughput. The mass resolution can be improved by narrowing the entrance and exit slits, however, at the cost of a reduced transmission.

\section{Deposition}

Figure 4 shows scanning tunneling pictures of a HOPG surface covered with (a) $\mathrm{Ce}_{0} \mathrm{C}_{60}$ and (b) $\mathrm{C}_{60}$ clusters, the

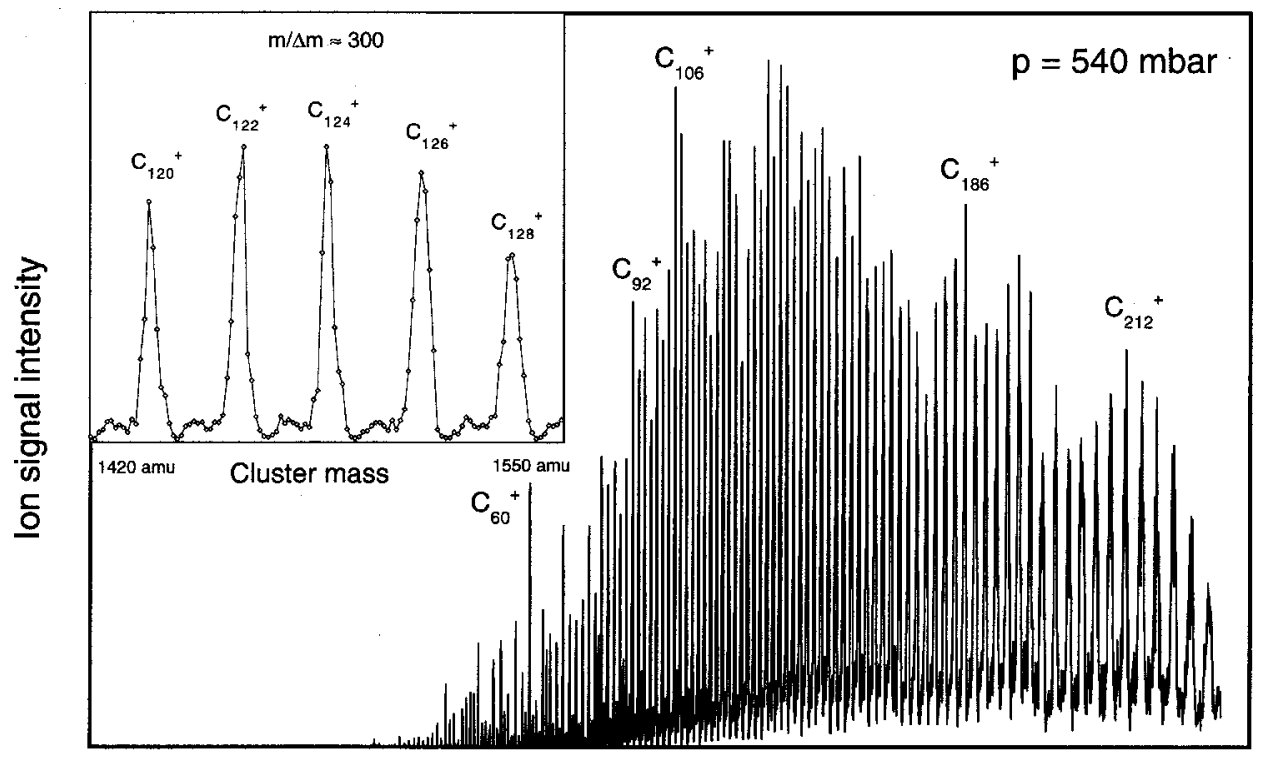

FIG. 3. Mass spectrum of pristine carbon cluster cations with masses up to $2600 \mathrm{amu}$, taken at a helium background pressure of 540 mbar. The inset shows the mass peaks of $\mathrm{C}_{120}{ }^{+}-\mathrm{C}_{128}{ }^{+}$. An analysis of the peak widths yields a mean resolution $m / \Delta m \approx 300$. 


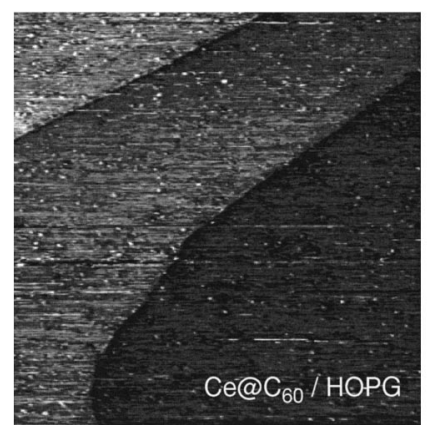

(a)

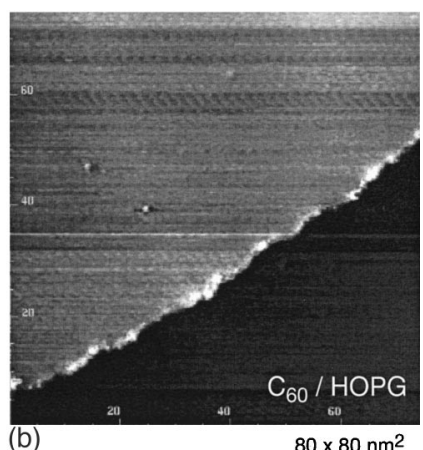

$80 \times 80 \mathrm{~nm}^{2}$
FIG. 4. STM pictures of (a) $\mathrm{Ce} @ \mathrm{C}_{60}$ and (b) $\mathrm{C}_{60}$ on $\mathrm{HOPG}$ at room temperature. Note the fairly uniform cluster distribution in (a).

latter in a low coverage region. ${ }^{18}$ The STM data have been measured at room temperature using a chemically etched tungsten tip. ${ }^{36}$ The tip was prepared for scanning tunneling spectroscopy measurements as described previously. ${ }^{37}$ This results in a somewhat reduced spatial resolution but is better suited for spectroscopic measurements. The clusters have been deposited for $5 \mathrm{~h}$ at an average ion current of $\sim 1 \mathrm{pA}$. The impact kinetic energy of the clusters was kept at 180 meV per atom, which is well below the limit for "soft landing." Since both the cluster source and the substrate have been kept at ground bias, the remaining kinetic energy during deposition results from the adiabatic expansion $(\sim 11 \mathrm{eV})$ of the clusters. Due to the large number of vibrational degrees of freedom this is a very low energy with respect to the one of thermally evaporated $\mathrm{C}_{60}\left(\sim 800^{\circ} \mathrm{C}\right)$ corresponding to a total excitation energy of about $16 \mathrm{eV}$. The background pressure during deposition has been $1 \times 10^{-8}$ mbar, mainly due to helium.

Terraces of the HOPG substrate are visible in the survey scans, separated from each other by monoatomic step edges. The clusters are the bright spots having an apparent diameter of about $30 \AA$. They show a fairly uniform distribution as expected from a statistical deposition from the gas phase [Fig. 4(a)]. The clusters do not migrate at room temperature as several consecutive STM pictures show an unchanged topology. Island formation has not been observed, and a decoration of step edges does not appear. From this we conclude that the interaction between the clusters and the HOPG substrate is fairly strong. The maximum height of the clusters is $6.7 \AA$, which is close to the diameter of free $\mathrm{C}_{60}$, confirming that the clusters survive the deposition process indestructed. Note that the height of structures on a surface can be determined quite accurately by STM, whereas the lateral size appears enlarged due to a convolution of the cluster shape and the tip geometry. The increased lateral size of individual clusters in STM scans has been observed previously on samples of $\mathrm{C}_{60}$ on $\mathrm{Si}(100)$ that have been prepared by thermal evaporation of fullerite. ${ }^{38}$ The density of clusters on the surface is one per $8 \times 8 \mathrm{~nm}^{2}$ as revealed by counting the spots on the surface. This value is in rough coincidence with the one expected from the measured ion current during deposition. Scanning tunneling spectroscopy data taken at the sample shown in Fig. 4(b) are in agreement with previous investigations that have been performed using samples of thermally evaporated $\mathrm{C}_{60}$ molecules. ${ }^{19,21}$ Moreover, an overall agreement between the measured peak positions and calculated local-density-approximation eigenvalues has been found.

\section{ACKNOWLEDGMENTS}

I. Wirth, G. Kann, and S. Eisebitt are gratefully acknowledged for providing the STM measurements. The authors are also grateful for the technical support by $\mathrm{H}$. Pfeifer and J. Lauer. This work has been supported by the Sonderforschungsbereich 341 of the Deutsche Forschungsgemeinschaft.

${ }^{1}$ W. A. deHeer, Rev. Mod. Phys. 65, 611 (1993).

${ }^{2}$ R. Busani, M. Folkers, and O. Cheshnovsky, Phys. Rev. Lett. 81, 3836 (1998).

${ }^{3}$ E. A. Rohlfing, D. M. Cox, and A. Kaldor, J. Chem. Phys. 81, 3322 (1984).

${ }^{4}$ H. Gohlich, T. Lange, T. Bergmann, and T. P. Martin, Mod. Phys. Lett. B 5, 101 (1991).

${ }^{5}$ D. E. Clemmer, J. M. Hunter, K. B. Shelimov, and M. F. Jarrold, Nature (London) 372, 248 (1994).

${ }^{6}$ K. B. Shelimov, D. E. Clemmer, and M. F. Jarrold, J. Phys. Chem. 99, 11376 (1995).

${ }^{7}$ H. Wu, S. R. Desai, and L.-S. Wang, Phys. Rev. Lett. 76, 212 (1996).

${ }^{8}$ H. Kietzmann, J. Morenzin, P. S. Bechthold, G. Ganteför, W. Eberhardt, D.-S. Yang, P. A. Hackett, R. Fournier, T. Pang, and C. Chen, Phys. Rev. Lett. 77, 4528 (1996).

${ }^{9}$ U. Heiz, A. Sanchez, S. Abbet, and W.-D. Schneider, J. Am. Chem. Soc. 121, 3214 (1999).

${ }^{10}$ S. Kakar, O. Björneholm, J. Weigelt, A. R. B. de Castro, L. Tröger, R. Frahm, T. Möller, A. Knop, and E. Rühl, Phys. Rev. Lett. 78, 1675 (1997).

${ }^{11}$ W. Eberhardt, P. Fayet, D. M. Cox, Z. Fu, A. Kaldor, R. Sherwood, and D. Sondericker, Phys. Rev. Lett. 64, 780 (1990).

${ }^{12}$ J. H. Weaver, Y. Chai, G. H. Kroll, C. Jin, T. R. Ohno, R. E. Haufler, T. Guo, J. M. Alford, J. Conceicao, L. P. F. Chibante, A. Jain, G. Palmer, and R. E. Smalley, Chem. Phys. Lett. 190, 460 (1992).

${ }^{13}$ B.-B. Liu, G.-T. Zou, H.-B. Yang, S. Yu, J.-S. Lu, Z.-Y. Liu, S.-Y. Liu, and W.-G. Xu, J. Phys. Chem. Solids 58, 1873 (1997).

${ }^{14}$ T. Pichler, M. S. Golden, M. Knupfer, J. Fink, U. Kirbach, P. Kuran, and L. Dunsch, Proceedings of the International Winterschool on Electronic Properties of Novel Materials, Kirchberg, Austria (World Scientific, Singapore, 1997).

${ }^{15}$ M. Krause, P. Kuran, U. Kirbach, and L. Dunsch, Carbon 37, 113 (1999).

${ }^{16}$ B. Kessler, A. Bringer, S. Cramm, C. Schlebusch, W. Eberhardt, S. Suzuki, Y. Achiba, F. Esch, M. Barnaba, and D. Cocco, Phys. Rev. Lett. 79, 2289 (1997).

${ }^{17}$ J. N. O'Shea, J. Schnadt, S. Andersson, L. Patthey, S. Rost, A. Giertz, B. Brena, J.-O. Forsell, A. Sandell, O. Björneholm, P. A. Brühwiler, and N. Mårtensson, J. Chem. Phys. 113, 9233 (2000).

${ }^{18}$ R. Klingeler, G. Kann, I. Wirth, S. Eisebitt, P. S. Bechthold, M. Neeb, and W. Eberhardt, J. Chem. Phys. 115, 7215 (2001).

${ }^{19}$ X. Yao, T. G. Ruskell, R. K. Workman, D. Sarid, and D. Chen, Surf. Sci. 366, L743 (1996).

${ }^{20}$ P. G. Collins, J. C. Grossman, M. Cote, M. Ishigami, C. Piskoti, S. G. Louie, M. L. Cohen, and A. Zettl, Phys. Rev. Lett. 82, 165 (1999).

${ }^{21}$ A. W. Dunn, E. D. Svensson, and C. Dekker, Surf. Sci. 498, 237 (2002).

${ }^{22}$ A. Piednoir, E. Perrot, S. Granjeaud, A. Humbert, C. Chapon, and C. R. Henry, Surf. Sci. 391, 19 (1997).

${ }^{23}$ U. Heiz, F. Vanolli, L. Trento, and W.-D. Schneider, Rev. Sci. Instrum. 68, 1986 (1997).

${ }^{24}$ A. Bettac, L. Köller, V. Rank, and K. H. Meiwes-Broer, Surf. Sci. 402404, 475 (1998)

${ }^{25}$ T. Schlenker, Diploma thesis, Universität Konstanz, 2000.

${ }^{26}$ B. Kaiser, T. M. Bernhardt, and K. Rademann, Appl. Phys. A: Mater. Sci. Process. 66, S711 (1998).

${ }^{27}$ J. T. Lau, A. Achleitner, and W. Wurth, Surf. Sci. 467, L834 (2000).

${ }^{28}$ J. T. Lau, A. Achleitner, and W. Wurth, Chem. Phys. Lett. 317, 269 (2000).

${ }^{29}$ C. Felix, C. Sieber, W. Harbich, J. Buttet, I. Rabin, W. Schulze, and G. Ertl, Phys. Rev. Lett. 86, 2992 (2001). 
${ }^{30}$ R. Schaub, H. Jodicke, F. Brunet, R. Monot, J. Buttet, and W. Harbich, Phys. Rev. Lett. 86, 3590 (2001).

${ }^{31}$ B. von Issendorf and R. E. Palmer, Rev. Sci. Instrum. 70, 4497 (1999).

${ }^{32}$ M. Neeb, R. Klingeler, P. S. Bechthold, G. Kann, I. Wirth, S. Eisebitt, and W. Eberhardt, Appl. Phys. A: Mater. Sci. Process. 72, 289 (2001).

${ }^{33}$ D. E. Powers, S. G. Hansen, M. E. Geusic, A. C. Pulu, J. B. Hopkins, T. G. Dietz, M. A. Duncan, P. R. R. Langridge-Smith, and R. E. Smalley, J. Phys. Chem. 86, 2556 (1982).
${ }^{34}$ R. Klingeler, P. S. Bechthold, M. Neeb, and W. Eberhardt, J. Chem. Phys. 113, 1420 (2000).

${ }^{35}$ T. Kimura, T. Sugai, and H. Shinohara, Int. J. Mass. Spectrom. 188, 225 (1999).

${ }^{36}$ G. H. Kann, Ber. Forschungszent. Juelich 3834, D38 (2000).

${ }^{37}$ R. M. Feenstra, J. A. Stroscio, and A. P. Fein, Surf. Sci. 181, 295 (1986).

${ }^{38}$ D. Klyachko and D. M. Chen, Surf. Sci. 446, 98 (2000). 